\title{
Chelate and Pincer Carbene Complexes of Rhodium and Platinum Derived from Hexaphenylcarbodiphosphorane, $\mathrm{Ph}_{3} \mathrm{P}=\mathrm{C}=\mathrm{PPh}_{3}$
}

\author{
Kazuyuki Kubo ${ }^{\ddagger}$ Nathan D. Jones ${ }^{\dagger}{ }^{\text {Michael J. Ferguson, }},{ }^{\dagger, \#}$ Robert McDonald ${ }^{\dagger, \#}$ and \\ Ronald G. Cavell ${ }^{\dagger *}$ \\ ${ }^{\ddagger}$ Department of Chemistry, Graduate School of Science, Hiroshima University, Higashi- \\ Hiroshima, 739-8526 Japan \\ ${ }^{\dagger}$ Department of Chemistry, University of Alberta, Edmonton, AB, Canada T6G $2 G 2$ \\ ${ }^{\#} X$-ray Crystallographic Laboratory
}

\section{SUPPORTING INFORMATION}

\section{Experimental Details}

\section{a. General}

All manipulations were performed under an $\mathrm{N}_{2}$ atmosphere using standard Schlenk techniques. Solvents were dried over appropriate drying agents and degassed by several freeze-pump-thaw cycles prior to use. Hexaphenylcarbodiphosphorane $(\mathbf{1}),{ }^{1}\left[\left(\eta^{4}-\operatorname{cod}\right) \mathrm{RhCl}\right]_{2} \quad(\operatorname{cod}=1,5-$ cyclooctadiene $)^{2}$ and $\left[\mathrm{Me}_{2} \mathrm{Pt}\left(\mu-\mathrm{SMe}_{2}\right)\right]_{2}{ }^{3}$ were prepared by literature methods. All other reagents were used as received from commercial sources. All analytical techniques were conducted within the Department of Chemistry at the University of Alberta. Unless otherwise indicated, NMR spectra were recorded at ambient temperature using $\mathrm{C}_{6} \mathrm{D}_{6}$ solutions of the complexes on a Varian i400 spectrometer $\left(161.9 \mathrm{MHz}\right.$ for ${ }^{31} \mathrm{P}, 100.6 \mathrm{MHz}$ for $\left.{ }^{13} \mathrm{C}\right)$ and referenced to residual solvent 
proton $\left({ }^{1} \mathrm{H}\right)$, solvent carbon $\left({ }^{13} \mathrm{C}\right)$, or external $85 \% \mathrm{H}_{3} \mathrm{PO}_{4}$. All coupling constant values are given in $\mathrm{Hz} ; \mathrm{s}=$ singlet, $\mathrm{d}=$ doublet, $\mathrm{t}=$ triplet, $\mathrm{m}=$ multiplet, $\mathrm{p}=$ pseudo. Where known, superscripts denote the number of bonds through which the coupling is mediated. Elemental (CHN) analyses were performed using a Carlo Erba EA1108 elemental analyser by Ms. Darlene Mahlow.

\section{b. Syntheses}

Preparation of $\left(\eta^{4}-\operatorname{cod}\right) \mathbf{R h}\left[\eta^{2}-\mathbf{C}\left\{\mathbf{P P h}_{2}\left(\mathbf{C}_{6} \mathbf{H}_{4}\right)\right\}\left\{\mathbf{P P h}_{3}\right\}\right]$ (2). To a suspension of $\left[\left(\eta^{4}-\operatorname{cod}\right) \mathrm{RhCl}\right]_{2}$ (116 mg, $0.24 \mathrm{mmol})$ in toluene $(10 \mathrm{~mL})$ was added $1(509 \mathrm{mg}, 0.95 \mathrm{mmol})$, and the mixture was stirred overnight at room temperature (r.t.) The product was extracted in hot toluene and filtered to remove $\left[\mathrm{HC}\left(\mathrm{PPh}_{3}\right)\right] \mathrm{Cl}$. After the volatile components were removed under vacuum, the resulting yellow powder was washed several times with small volumes of ether, and then dried under reduced pressure to give $273 \mathrm{mg}(0.37 \mathrm{mmol}, 78 \%)$ of 2 . Anal. Calcd for $\mathrm{C}_{45} \mathrm{H}_{41} \mathrm{P}_{2} \mathrm{Rh}$ : C, 72.39; H, 5.53. Found: C, 72.60; H, 5.51\%. ${ }^{1} \mathrm{H}$ NMR: $\delta 1.90(\mathrm{~m}, 4 \mathrm{H}$, cod-CH$), 2.06(\mathrm{~m}, 2 \mathrm{H}$, cod- $\left.\mathrm{CH}_{2}\right), 2.29\left(\mathrm{~m}, 2 \mathrm{H}\right.$, cod- $\left.\mathrm{CH}_{2}\right), 4.29(\mathrm{~m}, 2 \mathrm{H}$, cod- $\mathrm{CH}), 4.37(\mathrm{~m}, 2 \mathrm{H}$, cod-CH), 6.82-8.15 (m, 29H, $\mathrm{PPh}_{3}$ and $\left.\mathrm{PPh}_{2}\left(\mathrm{C}_{6} \mathrm{H}_{4}\right)\right) .{ }^{31} \mathrm{P}\left\{{ }^{1} \mathrm{H}\right\} \mathrm{NMR}: \mathrm{d} 10.15\left(\mathrm{~d},{ }^{2} J_{\mathrm{PP}}=50.9, \mathrm{PPh}_{3}\right.$ or $\left.P \mathrm{Ph}_{2}\left(\mathrm{C}_{6} \mathrm{H}_{4}\right)\right), 12.40$ $\left(\mathrm{d},{ }^{2} J_{\mathrm{PP}}=50.9, \mathrm{PPh}_{3}\right.$ or $\left.P \mathrm{Ph}_{2}\left(\mathrm{C}_{6} \mathrm{H}_{4}\right)\right) .{ }^{13} \mathrm{C}\left\{{ }^{1} \mathrm{H}\right\} \mathrm{NMR}: \delta 15.31\left(\mathrm{ddd},{ }^{1} J_{\mathrm{CRh}}=32.0,{ }^{1} J_{\mathrm{CP}}=71.4\right.$, $\left.{ }^{1} J_{\mathrm{CP}}=90.9, \mathrm{PCP}\right), 31.15\left(\mathrm{~s}, \mathrm{cod}-\mathrm{CH}_{2}\right), 31.75\left(\mathrm{~s}, \mathrm{cod}-C \mathrm{H}_{2}\right), 75.12\left(\mathrm{~d},{ }^{1} J_{\mathrm{CRh}}=12.4, \mathrm{cod}-C \mathrm{H}\right), 86.72$ $\left(\mathrm{d},{ }^{1} J_{\mathrm{CRh}}=6.9\right.$, cod$\left.-C \mathrm{H}\right), 121.92\left(\mathrm{~d}, J_{\mathrm{CP}}=12.9, \mathrm{PPh}_{2}\left(C_{6} \mathrm{H}_{4}\right)\right), 127.66\left(\mathrm{~d}, J_{\mathrm{CP}}=11.5, \mathrm{PPh}_{3}\right), 127.78$ $\left(\mathrm{d}, J_{\mathrm{CP}}=11.7, \mathrm{PPh}_{2}\left(\mathrm{C}_{6} \mathrm{H}_{4}\right)\right), 128.58\left(\mathrm{~d}, J_{\mathrm{CRh}}=2.8, \mathrm{C}_{6} \mathrm{H}_{4}\right), 129.53\left(\mathrm{~d}, J_{\mathrm{CP}}=2.8, \mathrm{P} P h_{2}\left(\mathrm{C}_{6} \mathrm{H}_{4}\right)\right)$, $130.27\left(\mathrm{~d}, J_{\mathrm{CP}}=2.8, \mathrm{PPh}_{3}\right), 133.31\left(\mathrm{~d}, J_{\mathrm{CP}}=9.7, \mathrm{PPh}\left(\mathrm{C}_{6} \mathrm{H}_{4}\right)\right), 133.65\left(\mathrm{~d}, J_{\mathrm{CP}}=10.6\right.$, $\left.\mathrm{PPh}_{2}\left(C_{6} \mathrm{H}_{4}\right)\right), 134.14\left(\mathrm{~d}, J_{\mathrm{CP}}=9.7, \mathrm{PPh}_{3}\right), 134.44\left(\mathrm{dd},{ }^{1} J_{\mathrm{CP}}=86.1,{ }^{3} J_{\mathrm{CP}}=6.4, \mathrm{PPh}_{3}\right), 135.67(\mathrm{~d}$, $\left.J_{\mathrm{CP}}=18.4, \mathrm{C}_{6} \mathrm{H}_{4}\right), 137.00\left(\mathrm{~d}, J_{\mathrm{CP}}=73.2, \mathrm{PPh} h_{2}\left(\mathrm{C}_{6} \mathrm{H}_{4}\right)\right), 150.81\left(\mathrm{dd},{ }^{1} J_{\mathrm{CP}}=127.7, \mathrm{~J}_{\mathrm{CP}}=11.7\right.$, $\left.\mathrm{C}_{6} \mathrm{H}_{4}\right), 181.26\left(\mathrm{dd},{ }^{2} J_{\mathrm{CP}}=33.2,{ }^{1} J_{\mathrm{CRh}}=39.1, \mathrm{C}_{6} \mathrm{H}_{4}\right)$. Rectangular X-ray quality (orange) crystals 
of 2 were obtained by dissolving the complex in hot benzene, filtering and slowly cooling to r.t. and allowing the crystallization to proceed at that temperature.

Preparation of $\mathbf{H R h}\left(\mathrm{PMe}_{3}\right)_{2}\left[\eta^{3}-\mathbf{C}\left\{\mathbf{P P h}_{2}\left(\mathbf{C}_{6} \mathbf{H}_{4}\right)\right\}_{2}\right]$ (3). To a suspension of $\mathbf{2}$ (162 mg, 0.22 mmol) in toluene $(10 \mathrm{~mL})$ was added $\mathrm{PMe}_{3}(1 \mathrm{M}$ in toluene, $0.50 \mathrm{~mL}, 0.50 \mathrm{mmol})$, and the mixture was stirred overnight at r.t. The resulting orange-yellow solution was filtered, and volatile components were removed under reduced pressure to give a yellow powder. The powder was washed with a small amount of pentane several times and dried under vacuum to yield 146 mg (0.18 mmol, $85 \%)$ of 3. Anal. Calcd for $\mathrm{C}_{43} \mathrm{H}_{47} \mathrm{P}_{4} \mathrm{Rh}$ : C, 65.32; H, 5.99. Found: C, 65.42; $\mathrm{H}, 6.09 \% .{ }^{1} \mathrm{H}$ NMR: $\delta-12.80\left(\mathrm{~m},{ }^{1} J_{\mathrm{HRh}}={ }^{2} J_{\mathrm{HP}(\mathrm{Me})}=22.6,{ }^{3} J_{\mathrm{HP}(\mathrm{Ph})}=5.1,1 \mathrm{H}, \mathrm{Rh} H\right), 0.94(\mathrm{t}$, $\left.{ }^{2} J_{\mathrm{HP}(\mathrm{Me})}=2.5,18 \mathrm{H}, \mathrm{PCH} H_{3}\right), 6.93-8.24\left(\mathrm{~m}, 28 \mathrm{H}, \mathrm{PPh} h_{2}\left(\mathrm{C}_{6} H_{4}\right)\right) .{ }^{31} \mathrm{P}\left\{{ }^{1} \mathrm{H}\right\} \mathrm{NMR}: \delta-6.23\left(\mathrm{~d},{ }^{1} J_{\mathrm{PRh}}=\right.$ 108.9, $\left.P \mathrm{Me}_{3}\right), 8.56\left(\mathrm{~s}, P \mathrm{Ph}_{2}\left(\mathrm{C}_{6} \mathrm{H}_{4}\right)\right) .{ }^{13} \mathrm{C}\left\{{ }^{1} \mathrm{H}\right\}$ APT NMR: $\delta 6.97\left(\mathrm{tdd},{ }^{1} J_{\mathrm{CP}(\mathrm{Ph})}=90.9,{ }^{1} J_{\mathrm{CRh}}=\right.$ $\left.19.5,{ }^{2} J_{\mathrm{CP}(\mathrm{Me})}=5.4, \mathrm{PCP}\right), 19.88\left(\mathrm{td},{ }^{1} J_{\mathrm{CP}}=14.2,{ }^{2} J_{\mathrm{CRh}}=1.3, \mathrm{PCH}_{3}\right), 119.80\left(\mathrm{~d}, J_{\mathrm{CP}}=7.4, \mathrm{C}_{6} \mathrm{H}_{4}\right)$, $127.91\left(\mathrm{~d}, J_{\mathrm{CP}}=5.3, \mathrm{Ph}\right), 129.43(\mathrm{~s}, \mathrm{Ph}), 130.76\left(\mathrm{~d}, J_{\mathrm{CP}}=11.3, \mathrm{C}_{6} \mathrm{H}_{4}\right), 132.54\left(\mathrm{~d}, \mathrm{~J}_{\mathrm{CP}}=4.8, \mathrm{Ph}\right)$, $133.64\left(\mathrm{~d}, J_{\mathrm{CRh}}=10.3, \mathrm{C}_{6} \mathrm{H}_{4}\right), 139.91(\mathrm{~m}, \mathrm{Ph}), 145.38\left(\mathrm{~d}, J_{\mathrm{CP}}=8.2, \mathrm{C}_{6} \mathrm{H}_{4}\right), 149.37\left(\mathrm{~m}, \mathrm{C}_{6} \mathrm{H}_{4}\right)$, $189.55\left(\mathrm{~m}, \mathrm{C}_{6} \mathrm{H}_{4}\right)$. X-ray quality (orange) blocky- crystals of $\mathbf{3}$ were obtained by dissolving the complex in benzene at r.t., reducing the volume under reduced pressure and allowing crystallization to proceed at r.t.

Preparation of $\left[\left(\mathbf{M e}_{2} \mathbf{S}\right) \mathbf{P t}\left\{\eta^{3}-\mathbf{C}\left(\mathbf{P}\left(\mathbf{C}_{6} \mathbf{H}_{4}\right) \mathbf{P h}_{2}\right)_{2}\right\}\right]$ (4). To a suspension of $\left[\mathrm{Me}_{2} \mathrm{Pt}\left(\mu-\mathrm{SMe}_{2}\right)\right]_{2}(224$ $\mathrm{mg}, 0.39 \mathrm{mmol})$ in toluene $(4 \mathrm{~mL})$ was added $1(422 \mathrm{mg}, 0.79 \mathrm{mmol})$. Gas evolution $\left(\mathrm{CH}_{4}\right)$ was observed immediately. After overnight stirring, the resulting solution was filtered, and the volatile components were removed from the filtrate under reduced pressure to give a yellow 
powder. The powder was then washed with ether several times and dried under vacuum to give $510 \mathrm{mg}(0.64 \mathrm{mmol}, 83 \%)$ of 4. Anal. Calcd for $\mathrm{C}_{39} \mathrm{H}_{34} \mathrm{P} 2 \mathrm{PtS}: \mathrm{C}, 59.16 ; \mathrm{H}, 4.33$. Found: $\mathrm{C}$, 59.14; H, $4.40 \% .{ }^{1} \mathrm{H}$ NMR: $\delta 2.35\left(\mathrm{~s},{ }^{3} J_{\mathrm{HPt}}=33.8,6 \mathrm{H}, \mathrm{SCH}_{3}\right), 6.78-8.46\left(\mathrm{~m}, 28 \mathrm{H}, \mathrm{PPh}_{3}\right.$ and $\left.\mathrm{PPh}_{2}\left(\mathrm{C}_{6} \mathrm{H}_{4}\right)\right) .{ }^{31} \mathrm{P}\left\{{ }^{1} \mathrm{H}\right\}$ NMR: $\delta 30.41\left(\mathrm{~s},{ }^{2} J_{\mathrm{PPt}}=183.5\right) .{ }^{13} \mathrm{C}\left\{{ }^{1} \mathrm{H}\right\}$ APT NMR: $\delta 11.34\left(\mathrm{t}, \mathrm{J}_{\mathrm{CP}}=\right.$ 98.2, PCP), $21.86(\mathrm{~s}, \mathrm{SCH}), 122.23\left(\mathrm{t}, J_{\mathrm{CP}}=7.2, \mathrm{C}_{6} \mathrm{H}_{4}\right), 128.00\left(\mathrm{~d}, J_{\mathrm{CP}}=5.5, \mathrm{Ph}\right), 129.48(\mathrm{~s}$, $\left.\mathrm{C}_{6} \mathrm{H}_{4}\right), 129.65\left(\mathrm{t}, J_{\mathrm{CP}}=10.6, \mathrm{C}_{6} \mathrm{H}_{4}\right), 130.20(\mathrm{~s}, \mathrm{Ph}), 132.96\left(\mathrm{t}, J_{\mathrm{CP}}=4.8, \mathrm{Ph}\right), 134.77(\mathrm{~m}, \mathrm{Ph})$, $138.11\left(\mathrm{t}, J_{\mathrm{CP}}=9.1, \mathrm{C}_{6} \mathrm{H}_{4}\right), 154.24\left(\mathrm{~m}, \mathrm{C}_{6} \mathrm{H}_{4}\right), 172.88\left(\mathrm{~m}, \mathrm{C}_{6} \mathrm{H}_{4}\right)$.

\section{Previously-Reported Structures}

The structures of our previously-reported Pt(II) carbene complexes are given in Figure S1.
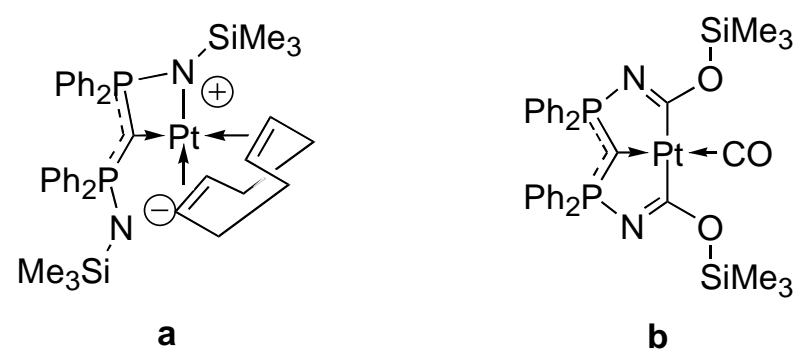

Figure S1. Our previously-reported $C, N$-chelate $(\mathbf{a})^{4}$ and $C, C, C$-pincer $(\mathbf{b})^{5}$ complexes of $\mathrm{Pt}(\mathrm{II})$ incorporating bis(phosphoranimine)-stabilized carbenes. 


\section{Computational details}

All calculations were performed at the density functional level using the Gaussian 98 suite of programs. 6 Becke's three-parameter exchange functional (B3) ${ }^{7}$ and Lee, Yang and Parr's correlation functional $(\mathrm{LYP})^{8}$ were used in conjunction with the LANL2DZ effective core potential basis set of Hay and Wadt. ${ }^{9}$ Orbital visualisation was conducted using the MOLDEN program. ${ }^{10}$ Pertinent results of these calculations are shown in Figure S2.
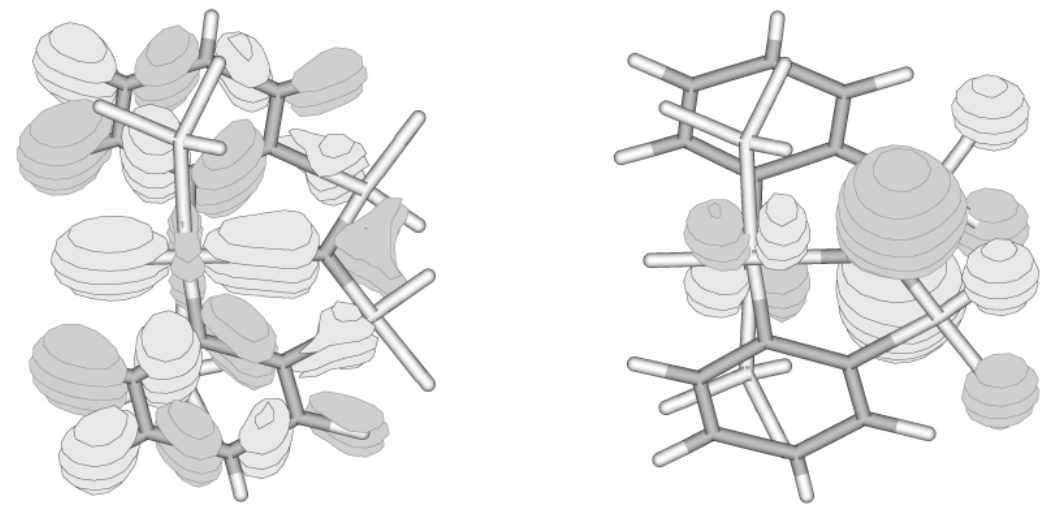

Figure S2. Representations of the calculated Rh-C $\sigma$-bonding molecular orbital (HOMO-14, left) and the HOMO of the model compound $\mathrm{HRh}\left(\mathrm{PH}_{3}\right)_{2}\left[\eta^{3}-\mathrm{C}\left\{\mathrm{H}_{2} \mathrm{P}\left(\mathrm{C}_{6} \mathrm{H}_{4}\right)\right\}_{2}\right]\left(3^{\prime}\right)$ 


\section{References}

(1) Ramirez, F.; Desai, N. B.; Hansen, B.; McKelvie, N. J. Am. Chem. Soc. 1961, 83, 3539-3540.

(2) Giordano, G.; Crabtree, R. H. Inorg. Synth. 1979, 19, 218-220.

(3) Scott, J. D.; Puddephatt, R. J. Organometallics 1983, 2, 1643-1648.

(4) Jones, N. D.; Lin, G.; Gossage, R .A.; McDonald, R.; Cavell, R. G. Organometallics 2003, 22, 2832-2841(erratum 5378).

(5) Lin, G.; Jones, N. D.; Gossage, R. A.; McDonald, R.; Cavell, R. G. Angew. Chem., Int. Ed. 2003, 42, 4054-4057.

(6) Gaussian 98, Revision A.11.4, Frisch, M. J.; Trucks, G. W.; Schlegel, H. B.; Scuseria, G. E.; Robb, M. A.; Cheeseman, J. R.; Zakrzewski, V. G.; Montgomery Jr, J. A.; Stratmann, R. E.; Burant, J. C.; Dapprich, S.; Millam, J. M.; Daniels, A. D.; Kudin, K. N.; Strain, M. C.; Farkas, O.; Tomasi, J.; Barone, V.; Cossi, M.; Cammi, R.; Menucci, B.; Pomelli, C.; Adamo, C.; Clifford, S.; Ochterski, J.; Petersson, G. A.; Ayala, P. Y.; Cui, Q.; Morokuma, K.; Rega, N.; Salvador, P.; Dannenberg, J. J.; Malick, D. K.; Rabuck, A. D.; Raghavachari, K.; Foresman, J. B.; Cioslowski, J.; Ortiz, J. V.; Baboul, G.; Stefanov, B. B.; Liu, G.; Liashenko, A.; Piskorz, P.;

Komaromi, I.; Gomperts, R.; Martin, R. L.; Fox, D. J.; Keith, T.; Al-Laham, M. A.; Peng, C. Y.; Nanayakkara, A.; Challacombe, M.; Gill, P. M. W.; Johnson, B. G.; Chen, W.; Wong, M. W.; Andres, J. L.; Gonzalez, C.; Head-Gordon, M.; Replogle, E. S.; Pople, J. A. Gaussian, Inc., Pittsburgh PA, 2002.

(7) Becke, A. D. J. Chem. Phys. 1993, 98, 5648-5642.

(8) Lee, C.; Yang, W.; Parr, R. G. Phys. Rev. 1998, B37, 785-789.

(9) Hay, P. J.; Wadt, W. R. J. Chem. Phys. 1985, 82, 270-283.

(10) Schaftenaar, G., Molden 3.9, University of Nijmegen, Nijmegen, The Netherlands, 2003. 